\title{
Therapeutic Development Paths for Cognitive Impairment in Parkinson's Disease: Report of a Regulatory Roundtable
}

\author{
Jamie Eberling $^{\mathrm{a}, *}$, Lona Vincent ${ }^{\mathrm{a}}$, Jennifer G. Goldman ${ }^{\mathrm{b}}$, Daniel Weintraub ${ }^{\mathrm{c}}$, Jaime Kulisevsky ${ }^{\mathrm{d}}$, \\ Connie Marras ${ }^{\mathrm{e}}$, Glenn Stebbins ${ }^{\mathrm{b}}$ and Karl Kieburtz ${ }^{\mathrm{f}}$ \\ a Michael J. Fox Foundation for Parkinson's Research, New York, NY, USA \\ ${ }^{\mathrm{b}}$ Rush University Medical Center, Chicago, IL, USA \\ ${ }^{\mathrm{c}}$ University of Pennsylvania, Philadelphia, PA, USA \\ ${ }^{\mathrm{d}}$ Sant Pau Hospital, Barcelona, Spain \\ ${ }^{\mathrm{e}}$ University of Toronto, Toronto, Canada \\ ${ }^{\mathrm{f}}$ University of Rochester, Rochester, NY, USA
}

\begin{abstract}
Cognitive impairment is a common occurrence in Parkinson's disease (PD), although the severity and specific presentation varies across patients. Initial deficits, including mild cognitive impairment (PD-MCI), may remain stable or in many cases, may progress over variable lengths of time to Parkinson's disease dementia (PDD). As there are currently no marketed treatments for milder forms of cognitive impairment, an opportunity exists to define the path for therapeutic development in this area. In the absence of a well-defined path for the approval of therapies that target PD-MCI, pharmaceutical companies are unlikely to pursue this indication. In order to move forward and improve the quality of life for PD patients, it is imperative for the field to have consensus on the definition of PD-MCI, the best instruments to measure cognitive decline, and a strategy for future clinical trials.
\end{abstract}

Keywords: Parkinson's, cognition, FDA, clinical trial

As an initial approach to address existing obstacles and gather the perspective of thought leaders in the field, in April 2013 the Michael J. Fox Foundation for Parkinson's Research (MJFF) organized the "Regulatory Roundtable for Cognitive Impairment in PD". In attendance were representatives from MJFF, industry, the Alzheimer's disease (AD) community, International Parkinson and Movement Disorders Society (IPMDS; formerly the Movement Disorders Society (MDS)), National Institute for Neurological Disorders and Stroke (NINDS), Parkinson's Action Network (PAN), Parkinson's Progression Markers Initiative (PPMI), Coalition Against Major Diseases

${ }^{*}$ Correspondence to: Jamie Eberling, PhD, 498 Seventh Ave, 18th Floor, New York, NY 10018, USA. Tel.: +1 212509 0995/Ext. 278; E-mail: JEberling@michaeljfox.org.
(CAMD), 16 representatives from the U.S. Food and Drug Administration (FDA), and key opinion leaders on cognitive impairment in PD. The goal of the meeting was to identify the regulatory requirements for pursuing a therapeutic indication for cognitive impairment in PD, focusing on pre-dementia stages. The discussion concentrated on the diagnostic criteria for PD-MCI, existing assessment and outcome measures, and short-term and long-term strategies for therapeutic development.

\section{DEFINING AND DIAGNOSING PD-MCI}

Estimating the prevalence of PD-MCI has been challenging due to the heterogeneous criteria used to diagnose and define the condition. Recent reviews 
report a mean prevalence of $27 \%$, ranging from $19 \%$ to $38 \%$ [1]. To aid in defining the condition, the recent publication of MDS PD-MCI diagnostic criteria provide a uniform definition of PD-MCI that can be readily used in both clinical and research settings. A common definition of PD-MCI to be utilized by patients, clinicians, and researchers is necessary to help identify the clinical characteristics of PD-MCI, the best predictors of conversion to PDD, and the effects of PD-MCI on quality of life and daily functioning. In addition, a uniform definition is critical for defining patient populations for inclusion in research studies, and for allowing clinicians, researchers, and patients and caregivers to communicate among each other and across settings. These criteria aim to define a group of PD patients who report cognitive decline compared with their premorbid state, exhibit cognitive deficits based on normative data, but do not have functional deficits significant enough to meet criteria for PDD. For many patients, the exact percentage of whom still needs to be determined, the "MCI" stage may represent a transitional point between normal cognition and dementia, and thus a potential harbinger of PDD. While many patients with PD-MCI convert to dementia, PDMCI may have a variable course such that for some patients, it remains a rather static condition, while in others, follow-up testing demonstrates improvement $[2,3]$. Determining the factors that govern this heterogeneity nature and course of PD-MCI, including its different subtypes, will be important factors in developing therapeutic interventions and designing clinical trials in PD-MCI [4-6].

The MDS PD-MCI diagnostic criteria represent a valuable tool for clinical practice and provide a uniform definition of the clinical syndrome, but are being, and continue to need to be, applied and validated in a range of clinical and research settings. Operational issues such as how to elicit and define a decline in cognitive ability from the patient or informant, and what a level of subtle functional impairment related to cognitive changes is acceptable to still fulfill criteria for MCI (rather than dementia) still need to be defined in order to ensure that a clearly-defined population is being studied. While a 'subjective complaint' by the patient or informant was a cornerstone of early MCI criteria [7], this aspect is less critical in the MDS PD-MCI criteria as decline observed by the clinician can suffice. Methods to elicit cognitive complaints are discussed in Marras et al. [8]. Measurement strategies for functional impairment are discussed in more detail below, and will be a key aspect of demonstrating therapeutic efficacy. From the regulatory perspective, it is the opinion of the authors that the FDA may be willing to consider an indication of PD-MCI without reference to the underlying neurobiological substrate, as was done when recognizing PDD in the context of approval for rivastigmine. This implies that clinical testing and trials can be initiated while the field pursues additional research on defining PD-MCI. This also implies that though having standardized diagnostic criteria will be valuable for clinical research, to receive drug approval sponsors will only be required to demonstrate that their patient population has a cognitive deficit, whether proven related to the underlying PD or not, and that this deficit is improved by the therapy under development.

\section{ASSESSING PD-MCI - COGNITION SCALES}

Successful clinical trials depend on outcome measures that are able to accurately reflect change in cognition due to the therapeutic agent or intervention. For some time it has been recognized that frequently used global cognitive instruments, such as the Mini-Mental State Examination (MMSE) [9] and the Alzheimer's Disease Assessment Scale-cognitive subscale (ADAS-cog), [10] do not map well onto the mild cognitive deficits reported to occur in many PD patients due to their focus on "cortical" deficits and their development for non-PD populations. As a result, several global cognitive assessment instruments have been developed or undergone validation for use in PD, including the Parkinson's DiseaseCognitive Rating Scale (PD-CRS), [11] the Parkinson Neuropsychometric Dementia Assessment (PANDA), [12] the Scales for Outcomes of Parkinson's DiseaseCognition (SCOPA-COG), [13] the Dementia Rating Scale-2 (DRS-2), [14] and the Montreal Cognitive Assessment (MoCA) $[15,16]$. In addition, a plethora of individual neuropsychological tests have been used in PD to specifically assess executive, memory, attention, visuospatial and language domain abilities, often in different combinations to create a battery of tests. This heterogeneity of neuropsychological tests and batteries used in many studies of PD cognitive impairment has been another challenge in assessing PD-MCI.

All of the existing instruments have limitations, and none (other than the MoCA for screening purposes) has yet gained wide acceptance. In the short term, the FDA indicated that sponsors should focus on selecting the scale or domain that is most appropriate for the trial design and sample population and that they are not endorsing any particular scale for use in PD-MCI 
trials. This is consistent with the methodology of the MDS PD-MCI diagnostic criteria, which allows the cognitive evaluation to be conducted with a number of existing instruments. Though the case can be made that there remains a need for a consensus-driven neuropsychological assessment battery that is sensitive to early cognitive decline in PD and appropriate for use in all aspects of clinical research, more research is needed to probe the practicability of existing instruments in multicenter research before the field makes an investment in a new battery. In particular, there is a need for studies that assess the sensitivity of the various scales to change over time and in response to treatment. This type of information will be critical for trial design and power calculations but currently there are not enough data to know which PD-specific scales will be most effective.

\section{ASSESSING PD-MCI - THE ROLE OF FUNCTIONAL IMPAIRMENT}

The FDA indicated that they will require evidence of functional benefit in trials for PD-MCI. This will likely entail that a functional scale be used as a coprimary outcome to document that cognitive changes associated with an investigational drug are clinically relevant. Cognitive deficits identified in PD subjects diagnosed with PD-MCI may interfere significantly with their ability to implement higher-level instrumental activities of daily living (IADLs), although not as significantly as in PDD [17-19]. Functional decline may be assessed using generic activities of daily living (ADL) instruments that are commonly used in AD trials (e.g., the ADCS-ADL). However, PD-related motor impairment potentially biases the rating of some activities, [20] and the different cognitive profile of PD (e.g., more executive deficits than in AD) limit the utility and sensitivity of the AD-designed instruments [21].

There are two PD-specific functional instruments that have been developed to measure the impact of cognitive impairment. The PD-Cognitive Functional Rating Scale (PD-CFRS) is a validated and reliable 5minute questionnaire of 12 items that captures relevant functional changes related to early and late cognitive impairment in PD. It has shown good correlations with neuropsychological ratings and good capacity to detect meaningful changes over time. The Brief Penn Daily Activities Questionnaire (PDAQ), [22] a 15-item scale administered to knowledgeable informants, was designed to assess IADLs sensitive and specific to cognitive impairment in PD. Still unpublished, preliminary results from the validation process show that the PDAQ present good discriminative validity across stages of cognitive impairment. Additional studies are needed to determine if one or both of these functional scales could be useful for PD-MCI trials.

\section{PLANNING FOR CLINICAL TRIALS}

The design of clinical trials that are intended to measure short-term changes in cognitive function should be relatively simple; standard, parallel group or crossover designs. The latter may be particularly appealing in early, learning phase trials, because of the reduction in variability (which allows much smaller sample sizes) due to within-subject comparisons. Crossover designs have their own challenges (e.g., loss to follow up, sequence effects), but may still be useful in the early evaluation of agents intended to improve cognitive performance. On the other hand, assessing long-term change in cognitive performance, such as slowing cognitive decline or preventing the onset of dementia (i.e., disease-modifying effects), present a major design challenge, and biomarkers could play a critical role in such studies. It is premature to recommend specific biomarkers but ongoing research should help to determine those that are most useful and could include imaging (positron emission tomography and fluorodeoxyglucose, functional MRI, amyloid, and hopefully eventually alpha-synuclein) and cerebrospinal fluid, blood and genetic markers, the subject of a recent review [23]. As disentangling short-term improvements from long-term effects is difficult, a two-period design (e.g., delayed start or washout) can be considered. There are many inherent complexities in such designs (as seen with the ADAGIO study), [24] including the duration of the two study periods, avoiding dropouts, and methods to analyze the stability of differences observed at study completion. An attractive alternative may be a survival analysis, such as delay of time to onset of dementia or cognitive impairment. The main challenge of such 'survival' studies will be a rigorous, yet pragmatic, definition of these endpoints. Given the novel nature of this therapeutic endpoint, discussions of trial design with regulatory authorities in advance of the study will be essential.

\section{THE CURRENT THERAPEUTIC LANDSCAPE}

Many of the therapeutics that have been tested for PD-MCI and PDD are those that have been tested 
Box 1. Path Forward for Cognitive Impairment in PD

General Path Forward

- The FDA is open to an indication for mild cognitive impairment in PD

- For regulatory consideration, it may be appropriate to pursue a PD-MCI indication without reference to the underlying cause of impairment

- Drug approvals for an indication of PD-MCI will likely require a co-primary measure (a cognitive outcome measure and a functional measure)

Short Term Path:

For immediate drug development using existing scales and diagnostic criteria

Diagnostic Criteria

- Current MDS PD-MCI diagnostic criteria can be used to identify a patient population for clinical testing

- If a sponsor chooses not to use the criteria, they must demonstrate that the study participants have a cognitive deficit

Outcome Measures

- Existing cognitive scales are sufficient for use in clinical trials on PD-MCI

- Sponsors should select the outcome measure/scale most likely to demonstrate change in the patient population

- The scale does not have to be specific to Parkinson's nor does it need to be an established measure of response to treatment

- In addition to a cognitive scales, sponsors will be required to include a measure of function to demonstrate clinical meaningfulness

Long Term Path:

Includes efforts to validate diagnostic criteria and/or create new instruments

Diagnostic Criteria

- MDS will work to validate and modify the PD-MCI diagnostic criteria (where needed) so the field can have a standardized approach for identifying and classifying study participants

Outcome Measures

- The field should work at analyzing PD-MCI datasets across a number of research studies in order to identify the scale items and domains most sensitive to change

- If the existing scale instruments, either cognitive of functional, prove to be sub-optimal in clinical testing, the PD field could develop a new scale or a composite measure

Biomarkers

- The field should work toward the development of an imaging, fluid, or combination biomarker

for MCI and $\mathrm{AD}$, including cholinesterase inhibitors and memantine. This seems a reasonable strategy given the overlap in many of the cognitive symptoms although to date rivastigmine is the only approved drug for PDD, albeit modestly effective. Therapeutics currently being tested for PD-MCI include rasagiline, rivastigmine, droxidopa, and AVE8112, a PDE4 inhibitor (see clinicaltrials.gov NCT01723228, NCT01519271, NCT02066571, NCT01803945). In addition to pharmacological interventions, nonpharmacolocial interventions such as cognitive training, exercise programs, and brain stimulation techniques are being explored [25-27]. While this is a growing area of research there is a need for rigorous studies with appropriate control groups to definitively test these approaches. A clear regulatory path with established diagnostic criteria and outcome measures would likely increase interest in developing PD-MCI therapies.

\section{THE PATH FORWARD}

The pursuit of disease-modifying therapies for neurological diseases, including $\mathrm{PD}$, is the ultimate goal for drug developers in this therapeutic space, but the regulatory requirements for FDA approval and the expense and complexities of such clinical trials represent very high hurdles. Given the challenges in pursuing a disease-modifying approach for cognitive impairment, symptomatic therapies may represent an attractive way to move the path forward for drug development in PD-MCI at this point, and some of these may also prove to delay the time from PD-MCI to dementia. Symptomatic trials could be advantageous given their shorter duration compared with disease-modifying trials. Disease-modifying therapies, albeit likely more challenging to develop since requiring longer trials and more complicated study designs, remain the "holy grail", as they have the potential to impact on disease course. Slowing the progression of cognitive decline and thus forestalling or preventing the development of dementia would represent a tremendous advance in the management of patients with PD. The challenges posed do not mean that disease modification is not worth pursuing, but rather that symptomatic therapies may represent an easier initial target for advances in PD-MCI treatments.

The good news is that there is a path forward for therapeutic development for cognitive impairment in PD, though this path will likely continue to be modified as we advance our understanding of PD-MCI as a syndrome. In pursuit of drug development or clinical testing, the field will need to address the following challenges: [1] refining and validating the diagnostic criteria in order to identify the patients who are most likely to benefit from treatment, [2] understanding the prognostic significance of PD-MCI, including the trajectory of cognitive decline, [3] further developing current instruments, including those to measure functional 
impairment, and [4] discovering and validating cognition biomarkers, which likely will be critical for patient selection for clinical trials, as well as for objective and sensitive measures of change in cognition over time.

\section{CONFLICT OF INTEREST}

The authors have no conflict of interest to report.

The views expressed in this manuscript are solely those of the authors and do not represent any formal guidance from the Food and Drug Administration (FDA).

\section{REFERENCES}

[1] Litvan I, Goldman JG, Troster AI, Schmand BA, Weintraub D, Petersen RC, Mollenhauer B, Adler CH, Marter K, WilliamsGray CH, Aarsland D, Kulisevsky J, Rodriguez -Oroz MC, Burn DJ, Barker RA, \& Emre M (2012) Diagnostic criteria for mild cognitive impairment in Parkinson's disease: Movement Disorder Society Task Force guidelines. Mov Disord, 27, 349356.

[2] Petersen KF, Larsen JP, Tysnes OB, \& Alves G (2009) Prognosis of mild cognitive impairment in early Parkinson disease: The Norwegian ParkWest study. JAMA, 70, 580-586.

[3] Broeders M, de Bie RMA, Velseboer DC, Speelman JD, Muslimovic D, \& Schmand B (2013) Evolution of mild cognitive impairment in Parkinson disease. Neurology, 18, 346-352.

[4] Williams-Gray CH, Evans JR, Goris A, Foltynie T, Ban M, Robbins TW, Brayne C, Kolachana BS, Weinberger DR, Sawcer SJ, \& Barker RA (2009) The distinct cognitive syndromes of Parkinson's disease: 5 year follow-up of the CamPaIGN cohort. Brain, 132, 2958-2969.

[5] Williams-Gray CH, Mason SL, Evans JR, Foltynie T, Brayne C, Robbins TW, \& Barker RA (2013) The CamPaIGN study of Parkinson's disease: 10 year outlook in an incident population-based cohort. J Neurol Neurosurg Psychiatry, 84, 1258-1264.

[6] Goldman JG, Williams-Gray C, Barker RA, Duda JE, \& Galvin JE (2014) The spectrum of cognitive impairment in Lewy body diseases. Mov Disord, 29, 608-621.

[7] Petersen RC, Smith GE, Waring SC, Ivnik RJ, Tangalos EG, \& Kokmen E (1999) Mild cognitive impairment: Clinical characterization and outcome. Archives of Neurology, 56 303-308.

[8] Marras C, Troster AI, Kulisevsky J, \& Stebbins GT (2014) The tools of the trade: A state of the art "How to assess cognition" in the patient with Parkinson's disease. Mov Disord, 29, 584596.

[9] Folstein MF, Folstein SE, \& McHugh PR (1975) "Minimental state". A practical method for grading the cognitive state of patients for the clinician. J Psychiatr Res, 12, 189-198.

[10] Rosen WG, Mohs RC, \& Davis KL (1984) A new rating scale for Alzheimer's disease. Am J Psychiatry, 141, 1356-1364.

[11] Pagonabarraga J, Kulisevsky J, Llebaria G, García-Sánchez C, Pascual-Sedano B, \& Gironell A (2008) Parkinson's diseasecognitive rating scale: A new cognitive scale specific for Parkinson's disease. Mov Disord, 23, 988-1005.

[12] Kalbe E, Calabrese P, Kohn N, Hilker R, Riedel O, Wittchen HU, Dodel R, Otto J, Ebersbach G, \& Kessler J (2008) Screening for cognitive deficits in Parkinson's disease with the Parkinson neuropsychometric dementia assessment (PANDA) instrument. Parkinsonism Relat Disord, 14, 93-101.

[13] Marinus J, Visser M, Verwey NA, Verhey FRJ, Middelkoop HAM, Stiggelbout AM, \& Van Hilten JJ (2003) Assessment of cognition in Parkinson's disease. Neurology, 61, 1222-8.

[14] Matteau E, Dupre N, Langlois M, Provencher P, \& Simard M (2012) Clinical validity of the Mattis Dementia Rating Scale2 in Parkinson disease with MCI and dementia. J Geriatr Psychiatry Neurol, 25, 100-106.

[15] Hoops S, Nazem S, Siderowf AD, Duda JE, Xie SX, Stern $\mathrm{MB}, \&$ Weintraub D (2009) Validity of the MoCA and MMSE in the detection of MCI and dementia in Parkinson's disease. Neurology, 73, 1738-1745.

[16] Dalrymple-Alford JC, MacAskill MR, Nakas CT, Livingston L, Graham C, Crucian GP, Melzer TR, Kirwan J, Keenan R, Wells S, Porter RJ, Watts R, \& Anderson TJ (2010) The MoCA: Well-suited screen for cognitive impairment in Parkinson disease. Neurology, 75, 1717-25.

[17] Emre M, Aarsland D, Brown R, Burn DJ, Duyckaerts C, Mizuno Y, Broe GA, Cummings J, Olanow W, Poewe W, Quinn N, Sampaio C, Tolosa E, \& DuBois B (2007) Clinical diagnostic criteria for dementia associated with Parkinson's disease. Mov Disord, 22, 1689-1707; quiz 1837.

[18] Rosenthal E, Brennan L, Xie S, Hertig H, Milber J, Weintraub D, Karlawish J, \& Siderowf A (2010) Association between cognition and function in patients with Parkinson disease with and without dementia. Mov Disord, 25, 1170-1176.

[19] Kulisevsky J, Fernandez de Bobadilla R, Pagonabarraga J, Martinez-Horta S, Campolongo A, Garcia-Sanchez C, Pascual-Sedano B, Rebosa-Nogue R, \& Villa-Bonomo C (2013) Measuring functional impact of cognitive impairment: Validation of the Parkinson's disease cognitive functional rating scale. Parkinsonism Relat Disord, 19, 812-817.

[20] Shulman LM, Gruber-Baldini AL, Anderson KE, Fishman PS, Reich SG, \& Weiner WJ (2010) The clinically important difference on the unified Parkinson's disease rating scale. Arch Neurol, 67, 64-70.

[21] Feldman H, Sauter A, Donald A, Gelinis I, Gauthier S, Torfs K, Parys W, \& Mehnert A (2001) The disability assessment for dementia scale: A 12-month study of functional ability in mild to moderate severity Alzheimer disease. Alzheimer Dis Assoc Disord, 15, 89-95.

[22] Weintraub D, Shea JA, Rubright JD, Karlawish J, Rick J, Goldmann R, Hurtig H, Duda JE, Xie XX, \& Siderowf A (2013) Psychometric properties of the Brief Penn Daily Activities Questionnaire (PDAQ) for Parkinson's disease. (poster). MDS Congress, Sydney.

[23] Mollenhauer B, Rochester L, Chen-Plotkin A, \& Brooks D (2014) What can biomarkers tell us about cognition in Parkinson's disease? Mov Disord, 29, 622-633.

[24] Olanow CW, Rascol O, Hauser R, Feigin PD, Jankowic J, Lang A, Langston W, Melamed D, Poewe W, Stocchi F, Tolosa E, \& ADAGIO Study Investigators (2009) A double-blind, delayed-start trial of rasagiline in Parkinson's disease. $N$ Engl J Med, 361, 1268-78.

[25] Naismith SL, Mowszowski L, Diamond K, \& Lewis SJ (2013) Improving memory in Parkinson's disease: A healthy brain ageing cognitive training program. Mov Disord, 28, 10971103.

[26] Sinforiani E, Banchieri L, Zucchella C, Pacchetti C, \& Sandrini G (2004) Cognitive rehabilitation in Parkinson's disease. Arch Gerontolo Geriatr Suppl, 387-391.

[27] Hindle JV, Petrelli A, Clare L, \& Kalbe E (2013) Nonpharmacological enhancement of cognitive function in Parkinson's disease: A systematic review. Mov Disord, 28, 1034-1049. 